Ito, $\mathrm{K}$.

Osaka J. Math.

40 (2003), 639-657

\title{
SCHOTTKY GROUPS AND BERS BOUNDARY OF TEICHMÜLLER SPACE
}

\author{
Kentaro ITO \\ (Received July 15, 1999)
}

\section{Introduction}

We will show that every Kleinian group on the Bers boundary of the Teichmüller space is an algebraic limit of a sequence of Schottky groups. This claim has already proved by Otal [26], but our argument is completely different from that of Otal. In this paper, we extend the action of the mapping class group on a Bers slice to that on a wider class of Kleinian groups. We obtain a sufficient condition for the action of the mapping class group to be continuous at a given point and show that the orbit of every maximal cusp is dense in the Bers boundary.

Here, we explain the fundamental idea of extending the action of the mapping class group. Let $S$ be an oriented compact surface possibly with boundary $\partial S$, and let $T(S)$ be the Teichmüller space of complete hyperbolic structures on the interior of $S$ with finite area. Let $R(S)$ be the space of conjugacy classes $[\rho, G]$ of representations $\rho: \pi_{1}(S) \rightarrow G \subset \mathrm{PSL}_{2}(\mathbf{C})$ of $\pi_{1}(S)$ which map each component of the boundary $\partial S$ to parabolic elements. The subspace $Q F(S)$ of $R(S)$ consisting of discrete faithful representations whose images are quasi-Fuchsian groups is naturally identified with a product of Teichmüller spaces $T(S) \times T(\bar{S})$, where $\bar{S}$ denotes $S$ with its orientation reversed. We denote the canonical homeomorphism by

$$
Q: T(S) \times T(\bar{S}) \rightarrow Q F(S) .
$$

The mapping class group $\operatorname{Mod}(S)$ of $S$ naturally acts on $T(S)$ and $T(\bar{S})$ and hence on the Bers slice $B_{X}=Q(\{X\} \times T(\bar{S}))$ by

$$
Q(X, \bar{Y}) \mapsto Q(X, \sigma \bar{Y})
$$

for $(X, \bar{Y}) \in T(S) \times T(\bar{S})$ and $\sigma \in \operatorname{Mod}(S)$. A crucial observation is that the representation $Q(X, \sigma \bar{Y})$ has another description as follows;

$$
Q(X, \sigma \bar{Y})=Q\left(\sigma^{-1} X, \bar{Y}\right) \circ \sigma_{*}{ }^{-1},
$$

where $\sigma_{*}$ is the group isomorphism of $\pi_{1}(S)$ induced by $\sigma$. The right-hand side of the above equation suggests us a possibility to define the action of $\operatorname{Mod}(S)$ even when $\bar{Y}$ 
is pinched or degenerated. From this point of view, we extend the action of $\operatorname{Mod}(S)$ on the Bers slice $B_{X}$ to that on the subset $C_{X}$ of $R(S)$, which is called the extended Bers slice for $X$. A representation $[\rho, G]$ is an element of $C_{X}$ by definition if $G$ is a function group with an invariant component $\Omega_{0}(G)$ of the region of discontinuity $\Omega(G)$ which is covering $X \in T(S)$. We remark that the extended Bers slice $C_{X}$ contains the closure $\bar{B}_{X}$ of the Bers slice $B_{X}$ and that the restriction of the action to $\bar{B}_{X}$ coincides with the action defined by Bers in [4].

The set $\partial B_{X}=\bar{B}_{X}-B_{X}$ is called the Bers boundary. Bers [4] obtained a sufficient condition for the action of $\operatorname{Mod}(S)$ to be continuous at a give point in $\partial B_{X}$. On the other hand, it is known by that the action of $\operatorname{Mod}(S)$ on $\partial B_{X}$ is not always continuous (see Kerckhoff-Thurston [13]). In Section 3, we extend the Bers' result and obtain a sufficient condition for the action to be continuous at a given point in $C_{X}$ :

Corollary 3.2. Let $[\rho, G]$ be an element of $C_{X}$ such that all components of $\Omega(G) / G$ except for $X=\Omega_{0}(G) / G$ have no moduli of deformation. Then $\operatorname{Mod}(S)$ acts continuously at $[\rho, G]$.

It is known by McMullen [24] that the set of maximal cusps is dense in $\partial B_{X}$. Moreover, one can see that the set of maximal cusps in $\partial B_{X}$ decomposes into finitely many orbits under the action of $\operatorname{Mod}(S)$. In Section 5, we will show that each orbit is dense in $\partial B_{X}$ :

Theorem 5.5. For any maximal cusp in $\partial B_{X}$, its orbit under the action of $\operatorname{Mod}(S)$ is dense in $\partial B_{X}$.

Suppose that $S$ is a closed surface of genus $g \geq 2$. Now let $S_{X}$ be the subset of $C_{X}$ consisting of Schottky groups. In Section 6, we combine Corollary 3.2 (continuity at maximal cusps) and Theorem 5.5 (orbit density for maximal cusps) to obtain the following theorem:

Theorem 6.1. Suppose that $S$ is a closed surface of genus $\geq 2$. The set of accumulation points of $S_{X}$ contains the Bers boundary $\partial B_{X}$.

Remark. Gallo [10] has proved the claim of Theorem 6.1 for the case of genus 2. More generally, Otal [26] has already proved the claim of Theorem 6.1 for the case of any genus $\geq 2$ by using hyperbolic Dehn surgery theorem. In our proof, we will make use of Thurston's compactness theorem [28] instead of hyperbolic Dehn surgery theorem.

This paper is organized as follows: In Section 2, we give a definition of an extended Bers slice $C_{X}$ on which we shall define the action of the mapping class group. 
In Section 3, we obtain a sufficient condition for the action of $\operatorname{Mod}(S)$ to be continuous at a point $C_{X}$ (Corollary 3.2). In Section 5, we show that the orbit of every maximal cusp is dense in the Bers boundary $\partial B_{X}$ (Theorem 5.5). In Section 6, we prove our main theorem (Theorem 6.1) as a consequence of the preceding sections. In Sections 5 and 6, one of the crucial tools is Thurston's compactness theorem [28], which will be introduced in Section 4. In Section 7, we collect some properties of the set of Schottky groups $S_{X}$ which can be easily seen.

ACKnowledgements. The author would like to express his gratitude to Hiroshige Shiga for his useful suggestions. He also appreciates the referee's valuable comments on the previous manuscript of this paper.

\section{Preliminaries}

2.1. Teichmüller spaces and Kleinian groups. Let $S$ be a compact oriented surface of negative Euler characteristic possibly with boundary $\partial S$. The Teichmüller space $T(S)$ of $S$ is the set of equivalence classes of pairs $(f, X)$; where $X$ is a complete hyperbolic Riemann surface of finite area and $f: \operatorname{int}(S) \rightarrow X$ is an orientation preserving homeomorphism from the interior of $S$. Two pairs $\left(f_{1}, X_{1}\right)$ and $\left(f_{2}, X_{2}\right)$ are said to be equivalent if there is a conformal map $g: X_{1} \rightarrow X_{2}$ such that $g \circ f_{1}$ is isotopic to $f_{2}$. The equivalence class of $(f, X)$ is simply denoted by $X$.

The mapping class group $\operatorname{Mod}(S)$ is the group consisting of isotopy classes of orientation preserving homeomorphisms of $S$. The natural action of $\sigma \in \operatorname{Mod}(S)$ on $T(S)$ is given by

$$
\sigma(f, X)=\left(f \circ \sigma^{-1}, X\right) .
$$

We also consider the Teichmüller space $T(\bar{S})$ of $\bar{S}$, where $\bar{S}$ denotes $S$ with its orientation reversed. Then we have a canonical isomorphism $\iota: T(S) \rightarrow T(\bar{S})$ defined by $(f: S \rightarrow X) \mapsto(\bar{f}: \bar{S} \rightarrow \bar{X})$, where $\bar{X}$ is the reflection of $X$. The action of $\sigma \in$ $\operatorname{Mod}(S)=\operatorname{Mod}(\bar{S})$ on $T(\bar{S})$ is given by $\sigma(\bar{f}, \bar{X})=\left(\bar{f} \circ \sigma^{-1}, \bar{X}\right)$, so that $\sigma \circ \iota=\iota \circ \sigma$ is satisfied.

A Kleinian group $G$ is a discrete subgroup of $\operatorname{PSL}_{2}(\mathbf{C})$, which acts on the hyperbolic space $\mathbf{H}^{3}$ as isometries, and on the sphere at infinity $S_{\infty}^{2}=\hat{\mathbf{C}}$ as conformal automorphisms. The limit set of $G$ in $\hat{\mathbf{C}}$ is denoted by $\Lambda(G)$ and its complement $\hat{\mathbf{C}}-\Lambda(G)$, which is called the region of discontinuity of $G$, is denoted by $\Omega(G)$. A Kleinian group $G$ is called a function group if its region of discontinuity $\Omega(G)$ has an invariant component. If a function group $G$ has exactly two invariant components, it is called a quasi-Fuchsian group; otherwise, it has a unique invariant component.

2.2. Projective structures. For a given $X \in T(S)$, let $\Gamma_{X}$ be a Fuchsian group acting on the unit disc $\Delta=\{z \in \hat{\mathbf{C}}:|z|<1\}$ such that $X=\Delta / \Gamma_{X}$. A bounded holomorphic quadratic differential on $\Delta$ for $\Gamma_{X}$ is a holomorphic function $\varphi$ on $\Delta$ satis- 
fying $\varphi \circ \gamma\left(\gamma^{\prime}\right)^{2}=\varphi$ for any $\gamma \in \Gamma_{X}$ and $\|\varphi\|_{\infty}<\infty$, where $\|\varphi\|_{\infty}$ is the hyperbolic sup-norm of $\varphi$ defined by

$$
\|\varphi\|_{\infty}=\sup _{z \in \Delta}\left(1-|z|^{2}\right)^{2}|\varphi(z)| .
$$

We let $B_{2}\left(\Gamma_{X}\right)$ denote the set of bounded holomorphic quadratic differentials on $\Delta$ for $\Gamma_{X}$. Then $B_{2}\left(\Gamma_{X}\right)$ is a finite dimensional complex Banach space.

The developing map for $\varphi \in B_{2}\left(\Gamma_{X}\right)$ is a meromorphic local homeomorphism

$$
f_{\varphi}: \Delta \rightarrow \hat{\mathbf{C}}
$$

whose Schwarzian derivative $S\left(f_{\varphi}\right)$ is equal to $\varphi$. We always assume that the developing map $f_{\varphi}$ is normalized by the conditions $f_{\varphi}(0)=0, f_{\varphi}^{\prime}(0)=1$ and $f_{\varphi}^{\prime \prime}(0)=0$. Associated to the developing map $f_{\varphi}$, there is a group homomorphism

$$
\rho_{\varphi}: \Gamma_{X} \rightarrow \mathrm{PSL}_{2}(\mathbf{C})
$$

satisfying $f_{\varphi} \circ \gamma=\rho_{\varphi}(\gamma) \circ f_{\varphi}$ for all $\gamma \in \Gamma_{X}$. This group homomorphism $\rho_{\varphi}$ is said to be the holonomy representation for $\varphi$. We call the pair $\left(f_{\varphi}, \rho_{\varphi}\right)$ the (normalized) projective structure for $\varphi \in B_{2}\left(\Gamma_{X}\right)$. Then there is a bijective correspondence between the set of normalized projective structures and $B_{2}\left(\Gamma_{X}\right)$.

We denote by $\hat{C}\left(\Gamma_{X}\right)$ the subset of $B_{2}\left(\Gamma_{X}\right)$ consisting of elements $\varphi \in B_{2}\left(\Gamma_{X}\right)$ whose developing maps $f_{\varphi}$ are covering maps onto their images. For an element $\varphi$ of $\hat{C}\left(\Gamma_{X}\right)$, the holonomy image $G=\rho_{\varphi}\left(\Gamma_{X}\right)$ of $\Gamma_{X}$ is a function group (possibly with torsion) and $f_{\varphi}(\Delta)$ is an invariant component of $G$, which is denoted by $\Omega_{0}(G)$ (see [14] and [16] for more information). Moreover, we consider a subset $C\left(\Gamma_{X}\right)$ of $\hat{C}\left(\Gamma_{X}\right)$ as follows. An element $\varphi$ of $\hat{C}\left(\Gamma_{X}\right)$ is contained in $C\left(\Gamma_{X}\right)$ by definition if the developing map $f_{\varphi}: \Delta \rightarrow f_{\varphi}(\Delta)=\Omega_{0}(G) \subset \hat{\mathbf{C}}$ descends to a conformal isomorphism $X=\Delta / \Gamma_{X} \rightarrow f_{\varphi}(\Delta) / \rho_{\varphi}\left(\Gamma_{X}\right)=\Omega_{0}(G) / G$ where $G$ is the holonomy image $\rho_{\varphi}\left(\Gamma_{X}\right)$ of $\Gamma_{X}$. In summary,

$$
\begin{aligned}
& \hat{C}\left(\Gamma_{X}\right)=\left\{\varphi \in B_{2}\left(\Gamma_{X}\right) \mid f_{\varphi}: \Delta \rightarrow f_{\varphi}(\Delta) \subset \hat{\mathbf{C}} \text { is a covering map }\right\}, \\
& C\left(\Gamma_{X}\right)=\left\{\varphi \in \hat{C}\left(\Gamma_{X}\right) \mid X=\Delta / \Gamma_{X} \cong f_{\varphi}(\Delta) / \rho_{\varphi}\left(\Gamma_{X}\right)\right\} .
\end{aligned}
$$

If $\Gamma_{X}$ is maximal (i.e. there are no Fuchsian groups which properly contain $\left.\Gamma_{X}\right) C\left(\Gamma_{X}\right)$ coincides with $\hat{C}\left(\Gamma_{X}\right)$. Note that $\Gamma_{X}$ is maximal for almost every $X \in T(S)$. On the other hand, in [18], one can find examples of elements of $\hat{C}\left(\Gamma_{X}\right)$ but not of $C\left(\Gamma_{X}\right)$ for some $\Gamma_{X}$. For $\varphi \in C\left(\Gamma_{X}\right), G=\rho_{\varphi}\left(\Gamma_{X}\right)$ may have an elliptic element whose fixed points are not contained in the invariant component $\Omega_{0}(G)=f_{\varphi}(\Delta)$.

2.3. Extended Bers slices. A sequence of representations $\rho_{n}: \pi_{1}(S) \rightarrow \mathrm{PSL}_{2}(\mathbf{C})$ is said to converges algebraically to a representation $\rho: \pi_{1}(S) \rightarrow \operatorname{PSL}_{2}(\mathbf{C})$ if $\rho_{n}(g) \rightarrow$ 
$\rho(g)$ in $\mathrm{PSL}_{2}(\mathbf{C})$ for all $g \in \pi_{1}(S)$. The conjugacy class of a representation $\rho: \pi_{1}(S) \rightarrow$ $\mathrm{PSL}_{2}(\mathbf{C})$ with $\rho\left(\pi_{1}(S)\right)=G$ is denoted by $[\rho, G]$ or simply by $[\rho]$. Let $R(S)$ denote the space of conjugacy classes $[\rho]$ of irreducible representations $\rho: \pi_{1}(S) \rightarrow \operatorname{PSL}_{2}(\mathbf{C})$ such that $\rho(\gamma)$ is parabolic for every $\gamma \in \pi_{1}(\partial S)$. The space $R(S)$ is a complex manifold endowed with the topology of algebraic convergence.

It is known by Kra [15] that the map

$$
\text { hol: } B_{2}\left(\Gamma_{X}\right) \rightarrow R(S)
$$

defined by $\varphi \longmapsto\left[\rho_{\varphi}\right]$ is a holomorphic embedding, where $\left[\rho_{\varphi}\right]$ is the conjugacy class of the representation $\rho_{\varphi}: \pi_{1}(S) \cong \Gamma_{X} \rightarrow \mathrm{PSL}_{2}(\mathbf{C})$. (Here and hereafter, we frequently identify a representation of $\pi_{1}(S)$ with a representation of $\Gamma_{X}$.) For any $X \in T(S)$, we define subsets $\hat{C}_{X}$ and $C_{X}$ of $R(S)$ by

$$
\begin{aligned}
\hat{C}_{X} & =\operatorname{hol}\left(\hat{C}\left(\Gamma_{X}\right)\right), \\
C_{X} & =\operatorname{hol}\left(C\left(\Gamma_{X}\right)\right) .
\end{aligned}
$$

We call $C_{X}$ the extended Bers slice, on which we will define an action of the mapping class group.

The Bers slice $B_{X}$ is the subset of $C_{X}$ consisting of faithful representations whose images are quasi-Fuchsian groups. It is known by Bers [3] that the Bers slice $B_{X}$ can be identified with the Teichmüller space $T(S)$, and that it is relatively compact in $R(S)$. The set $\partial B_{X}=\bar{B}_{X}-B_{X}$ is called the Bers boundary, where $\bar{B}_{X}$ is the closure of $B_{X}$ in $R(S)$. Moreover, we denote by $\hat{B}_{X}$ the subset of $C_{X}$ consisting of faithful representations. It is conjectured that $\bar{B}_{X}=\hat{B}_{X}$ in Bers [3].

The following are the sets which we want to consider in this paper:

$$
B_{X} \subset \bar{B}_{X} \subseteq \hat{B}_{X} \subset C_{X} \subset \hat{C}_{X}
$$

ExAmple. In the case that $S$ is a closed surface, a typical example of an element of $C_{X}-\hat{B}_{X}$ is a Schottky group. A Kleinian group $G$ is a Schottky group if $G$ is torsion-free and if its Kleinian manifold $N_{G}=\left(\mathbf{H}^{3} \cup \Omega(G)\right) / G$ is homeomorphic to a handlebody $H_{g}$ of genus $g$. Let $G$ be a Schottky group which uniformizes $X$, that is $X=\Omega(G) / G=\partial N_{G}$, then the representation $\rho: \pi_{1}(S) \cong \pi_{1}\left(\partial N_{G}\right) \rightarrow G \cong \pi_{1}\left(N_{G}\right)$ induced by the inclusion map $\partial N_{G} \hookrightarrow N_{G}$ is an element of $C_{X}$ but not of $\hat{B}_{X}$.

Lemma 2.1. For any $X \in T(S), C_{X}$ is a compact subset of $R(S)$.

Proof. To show that $C_{X}=\operatorname{hol}\left(C\left(\Gamma_{X}\right)\right)$ is compact, it is enough to see that $C\left(\Gamma_{X}\right)$ is closed and bounded subset of $B_{2}\left(\Gamma_{X}\right)$. Since it is known by Kra and Maskit [18] that $\hat{C}\left(\Gamma_{X}\right)$ is a closed and bounded subset of $B_{2}\left(\Gamma_{X}\right)$, we only have to show that $C\left(\Gamma_{X}\right)$ is closed. Let $\varphi_{n} \in C\left(\Gamma_{X}\right)$ be a sequence converging to $\varphi \in \hat{C}\left(\Gamma_{X}\right)$. Let $f_{n}$ and 
$f$ be the developing maps corresponding to $\varphi_{n}$ and $\varphi$, respectively. Then $f_{n}$ converges to $f$ locally uniformly on $\Delta$. Suppose that the map $g: X \rightarrow f(\Delta) / \rho\left(\Gamma_{X}\right)$ induced by $f: \Delta \rightarrow f(\Delta)$ is not injective. Then there are two points $x, y \in X(x \neq y)$ such that $g(x)=g(y)$, and hence there are lifts $\tilde{x}, \tilde{y} \in \Delta$ of $x$ and $y$ such that $f(\tilde{x})=f(\tilde{y})$. Since $f_{n}(\tilde{x}) \rightarrow f(\tilde{x})$ and $f_{n}(\tilde{y}) \rightarrow f(\tilde{y})$, the hyperbolic distances $d_{n}$ between $f_{n}(\tilde{x})$ and $f_{n}(\tilde{y})$ in $f_{n}(\Delta)$ tend to 0 as $n \rightarrow \infty$. On the other hand, since $\varphi_{n} \in C\left(\Gamma_{X}\right)$, the maps $g_{n}: X \rightarrow f_{n}(\Delta) / \rho_{n}\left(\Gamma_{X}\right)$ induced by $f_{n}: \Delta \rightarrow f_{n}(\Delta)$ are conformal isomorphisms. Hence the hyperbolic distance between $x$ and $y$ on $X$ is equal to the hyperbolic distance between $g_{n}(x)$ and $g_{n}(y)$ on $f_{n}(\Delta) / \rho_{n}\left(\Gamma_{X}\right)$ which are less or equal to $d_{n}$. This contradicts to $x \neq y$.

2.4. Quasiconformal deformations. For a given Kleinian group $G$ with $\Omega(G) \neq$ $\emptyset$, a measurable function $\mu$ on $\widehat{\mathbf{C}}$ is called a Beltrami differential for $G$ if

$$
\mu(g(z)) \overline{g^{\prime}(z)}=\mu(z) g^{\prime}(z)
$$

holds for a.e. $z \in \widehat{\mathbf{C}}$ and for all $g \in G$. The space of all Beltrami differentials $\mu$ for $G$ whose essential sup-norm satisfying $\|\mu\|_{\infty}<1$ is denoted by $\operatorname{Belt}(G)_{1}$. For a $G$-invariant open set $U \subset \Omega(G)$, we denote by $\operatorname{Belt}(G, U)_{1}$ the subset of $\operatorname{Belt}(G)_{1}$ consisting of all elements with support in $U$. For $\mu \in \operatorname{Belt}(G, U)_{1}$, there is a unique quasiconformal homeomorphism

$$
w_{\mu}: \hat{\mathbf{C}} \rightarrow \hat{\mathbf{C}}
$$

satisfying $\left(w_{\mu}\right)_{\bar{z}} /\left(w_{\mu}\right)_{z}=\mu$ (a.e.) and fixing 0,1 and $\infty$. Two elements $\mu, \nu \in$ $\operatorname{Belt}(G, U)_{1}$ are equivalent (denoted by $\left.\mu \sim \nu\right)$ if $w_{\mu}$ and $w_{\nu}$ induce the same group isomorphism; that is, $w_{\mu} \circ g \circ\left(w_{\mu}\right)^{-1}=w_{\nu} \circ g \circ\left(w_{\nu}\right)^{-1}$ for all $g \in G$.

In the lest of this subsection, we restrict our attention to the following situation: For a given $X \in T(S)$, let $[\rho, G]$ be an element of $C_{X}$ and let $f: \Delta \rightarrow \Omega_{0}(G)$ be the developing map inducing the holonomy representation $\rho: \pi_{1}(S) \cong \Gamma_{X} \rightarrow G$.

We denote by $Q C([\rho])$ the space of quasi-conformal deformations of $[\rho]=[\rho, G]$ induced by elements of $\operatorname{Belt}(G)_{1}$. That is, $\left[\rho^{\prime}\right] \in R(S)$ is an element of $Q C([\rho])$ if $\rho^{\prime}(\gamma)=w_{\mu} \circ \rho(\gamma) \circ\left(w_{\mu}\right)^{-1}$ is satisfied for all $\gamma \in \pi_{1}(S)$ for some $\mu \in \operatorname{Belt}(G)_{1}$. This space $Q C([\rho])$ is identified with the quotient space $\operatorname{Belt}(G)_{1} / \sim$. Moreover, we denote by $Q C_{0}([\rho])$ the space of quasi-conformal deformations of $[\rho]=[\rho, G]$ induced by elements of $\operatorname{Belt}\left(G, \Omega_{0}(G)\right)_{1}$, that is $Q C_{0}([\rho])=\operatorname{Belt}\left(G, \Omega_{0}(G)\right)_{1} / \sim$.

Now let consider the case that $\rho$ is the identity representation id: $\pi_{1}(S) \cong \Gamma_{X} \rightarrow$ $\Gamma_{X}$ induced by the identity map id: $\Delta \rightarrow \Delta=\Omega_{0}\left(\Gamma_{X}\right)$. Then $\Omega\left(\Gamma_{X}\right)$ has exactly two component $\Delta=\Omega_{0}\left(\Gamma_{X}\right)$ and $\Delta^{*}=\{z \in \hat{\mathbf{C}}:|z|>1\}$. The space of quasiconformal deformations $Q C([\mathrm{id}])$ of [id] is said to be the quasi-Fuchsian space and denoted by $Q F(S)$. On the other hand, the quotient space $Q C_{0}([\mathrm{id}])=\operatorname{Belt}\left(\Gamma_{X}, \Delta\right)_{1} / \sim$ can be identified with the Teichmüller space $T(X) \cong T(S)$. Similarly, the quotient space $\operatorname{Belt}\left(\Gamma_{X}, \Delta^{*}\right)_{1} / \sim$ can be identified with the Teichmüller space $T(\bar{X}) \cong T(\bar{S})$, where 
$\bar{X}=\Delta^{*} / \Gamma_{X}$ is the reflection of $X$. It is the well known fact (cf. Bers [2]) that the map

$$
\operatorname{Belt}\left(\Gamma_{X}, \Delta\right) \times \operatorname{Belt}\left(\Gamma_{X}, \Delta^{*}\right) \rightarrow \operatorname{Belt}\left(\Gamma_{X}\right)
$$

defined by $(\mu, \nu) \mapsto \mu+\nu$ descends to the canonical homeomorphism

$$
Q: T(S) \times T(\bar{S}) \rightarrow Q F(S) .
$$

We remark that the Bers slice $B_{X}$ defined in Subsection 2.3 is equal to $Q(\{X\} \times T(\bar{S}))$.

Recall that the holonomy representation $\rho: \pi_{1}(S) \cong \Gamma_{X} \rightarrow G$ is induced by the developing map $f: \Delta \rightarrow \Omega_{0}(G)$. For $\mu \in \operatorname{Belt}\left(G, \Omega_{0}(G)\right)_{1}$, the pull-back $f^{*} \mu$ of $\mu$ by $f$ is defined by

$$
f^{*} \mu(z)=\mu \circ f(z) \frac{\overline{f^{\prime}(z)}}{f^{\prime}(z)} .
$$

Since $f$ descends to an isomorphism $\Delta / \Gamma_{X} \rightarrow \Omega_{0}(G) / G$, the map

$$
f^{*}: \operatorname{Belt}\left(G, \Omega_{0}(G)\right)_{1} \rightarrow \operatorname{Belt}\left(\Gamma_{X}, \Delta\right)_{1}
$$

defined by $\mu \mapsto f^{*} \mu$ is an isomorphism. This is the reason why we can define the action on $C_{X}$ but cannot on $\hat{C}_{X}$. We denote the inverse $\left(f^{*}\right)^{-1}$ of $f^{*}$ by $f_{*}: \operatorname{Belt}\left(\Gamma_{X}, \Delta\right)_{1} \rightarrow \operatorname{Belt}\left(G, \Omega_{0}(G)\right)_{1}$. Then, it was shown by Maskit [21] (see also Kra [17]) that the map $f_{*}: \operatorname{Belt}\left(\Gamma_{X}, \Delta\right)_{1} \rightarrow \operatorname{Belt}\left(G, \Omega_{0}(G)\right)_{1}$ descends to an unbranched covering map

$$
\underline{f_{*}}: T(S) \rightarrow Q C_{0}([\rho])
$$

with $\underline{f_{*}}(X)=[\rho]$. We use the notation

$$
\mathrm{qc}([\rho], Y)=\underline{f_{*}}(Y)
$$

for any $Y \in T(S)$. Then the representation $\mathrm{qc}([\rho], Y) \in Q C_{0}([\rho])$ can be regarded as the quasiconformal deformation of $[\rho]$ corresponding to the quasiconformal deformation $Y$ of $X$ in $T(S)$. Note that $\mathrm{qc}([\rho], X)=[\rho]$ and that $\mathrm{qc}([\rho], Y) \in C_{Y}$ for all $Y \in T(S)$.

2.5. The action of the mapping class group. Now we define the action of $\operatorname{Mod}(S)$ on $C_{X}$ by

$$
\sigma([\rho])=\mathrm{qc}\left([\rho], \sigma^{-1} X\right) \circ \sigma_{*}^{-1}
$$

for $[\rho] \in C_{X}$ and $\sigma \in \operatorname{Mod}(S)$, where $\sigma_{*}$ is the group isomorphism of $\pi_{1}(S)$ induced by $\sigma$. Since $\left[\rho^{\prime}\right] \circ \sigma_{*}^{-1} \in C_{\sigma Y}$ for any $\left[\rho^{\prime}\right] \in C_{Y}$ and $Y \in T(S)$, one can see that $\sigma([\rho])$ also contained in $C_{X}$. 


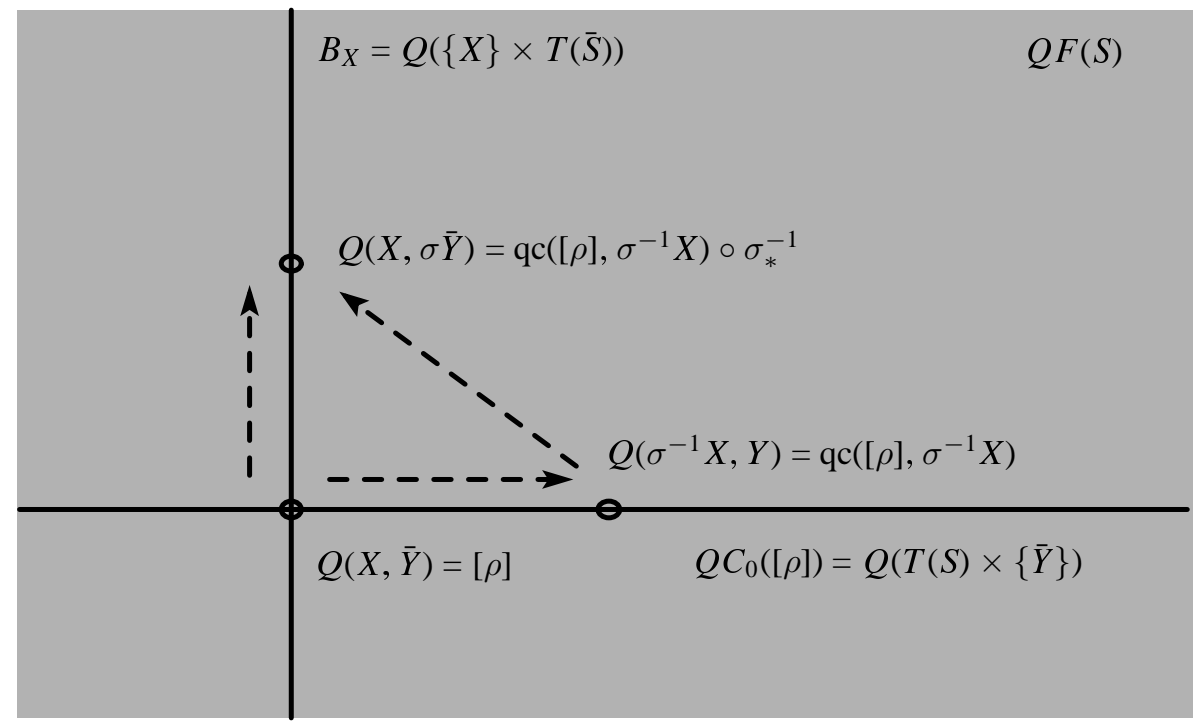

Fig. 1. The action of the mapping class group

Let take $[\rho] \in C_{X}$ and $\sigma \in \operatorname{Mod}(S)$ and put $\left[\rho^{\prime}\right]=\sigma([\rho])$. Then $\operatorname{ker} \rho^{\prime}=\sigma_{*}(\operatorname{ker} \rho)$ is satisfied. Therefore, if $\operatorname{ker} \rho \neq \sigma_{*}(\operatorname{ker} \rho), \sigma([\rho])$ is not a quasiconformal deformation of $[\rho]$ and hence $\sigma([\rho])$ is not contained in the connected component of $C_{X}$ containing $[\rho]$.

Here we explain that the action of $\operatorname{Mod}(S)$ on the Bers slice $B_{X}\left(\subset C_{X}\right)$ defined above coincides with the action on the Teichmüller space $T(\bar{S})$ under the identification $B_{X}=T(\bar{S})$ (see Fig. 1). The mapping class group $\operatorname{Mod}(S)$ acts on $B_{X}=$ $Q(\{X\} \times T(\bar{S}))$ by $Q(X, \bar{Y}) \mapsto Q(X, \sigma \bar{Y})$ for $Q(X, \bar{Y}) \in B_{X}$ and $\sigma \in \operatorname{Mod}(S)$. Now we put $[\rho]=Q(X, \bar{Y})$ and are going to show that $Q(X, \sigma \bar{Y})=\sigma([\rho])$. By definition, $\sigma([\rho])=\mathrm{qc}\left([\rho], \sigma^{-1} X\right) \circ \sigma_{*}^{-1}=Q\left(\sigma^{-1} X, \bar{Y}\right) \circ \sigma_{*}^{-1}$. Since $Q(\sigma X, \sigma Y)=Q(X, Y) \circ \sigma_{*}^{-1}$ holds for any $(X, \bar{Y}) \in T(S) \times T(\bar{S})$, one obtain the desired equation $Q(X, \bar{Y})=$ $Q\left(\sigma^{-1} X, \bar{Y}\right) \circ \sigma_{*}^{-1}$.

\section{Continuity of the action of the mapping class group}

In this section, we obtain a sufficient condition for $[\rho] \in C_{X}$ so that the action of $\operatorname{Mod}(S)$ at $[\rho]$ is continuous. The same result, where $C_{X}$ is replaced by $\hat{B}_{X}$, was obtained by Bers [4].

We first show the continuity for the change of base points.

Proposition 3.1. Let $[\rho, G]$ be an element of $C_{X}$ such that all components of $\Omega(G) / G$ except for $X=\Omega_{0}(G) / G$ have no moduli of deformation. Then the following holds: If $\left[\rho_{n}\right] \rightarrow[\rho]$ in $C_{X}$ then $\mathrm{qc}\left(\left[\rho_{n}\right], Y\right) \rightarrow \mathrm{qc}([\rho], Y)$ in $C_{Y}$ for all $Y \in T(S)$. 
Proof. Let $\varphi_{n}$ and $\varphi$ be elements in $C\left(\Gamma_{X}\right)$ such that $\operatorname{hol}\left(\varphi_{n}\right)=\left[\rho_{n}\right]$ and $\operatorname{hol}(\varphi)=$ [ $\rho$ ], respectively. Since $C\left(\Gamma_{X}\right)$ is compact and the map hol: $B_{2}\left(\Gamma_{X}\right) \rightarrow R(S)$ is injective, $\varphi_{n} \rightarrow \varphi$ in $C\left(\Gamma_{X}\right)$. Let $\left(f_{n}, \rho_{n}\right)$ and $(f, \rho)$ be normalized projective structures for $\varphi_{n}$ and $\varphi$, respectively. Then $f_{n}$ converges to $f$ locally uniformly on $\Delta$. Let $\mu \in \operatorname{Belt}\left(\Gamma_{X}, \Delta\right)_{1}$ be a representative of $Y \in T(S)=\operatorname{Belt}\left(\Gamma_{X}, \Delta\right)_{1} / \sim$. We may assume that $\mu$ is continuous function on $\Delta$. Put $\hat{\mu}_{n}=\left(f_{n}\right)_{*} \mu \in \operatorname{Belt}\left(G_{n}, \Omega_{0}\left(G_{n}\right)\right)_{1}$ and $\hat{\mu}=f_{*} \mu \in \operatorname{Belt}\left(G, \Omega_{0}(G)\right)_{1}$, where $G_{n}=\rho_{n}\left(\pi_{1}(S)\right)$ and $G=\rho\left(\pi_{1}(S)\right)$. Since $\left\{w_{\hat{\mu}_{n}}\right\}$ fix 0,1 and $\infty$ and their dilatations are uniformly bounded, it has a subsequence (which we denote by the same symbol) $\left\{w_{\hat{\mu}_{n}}\right\}$ converging uniformly to some quasiconformal homeomorphism $w_{\infty}$ of $\hat{\mathbf{C}}$. Since the representatives of $\operatorname{qc}\left(\left[\rho_{n}\right], Y\right)$ are induced by $w_{\hat{\mu}_{n}} \circ f_{n},\left\{\mathrm{qc}\left(\left[\rho_{n}\right], Y\right)\right\}$ converges algebraically to the conjugacy class of the representation induced by $w_{\infty} \circ f$. Therefore, we have only to show that $w_{\infty}$ and $w_{\hat{\mu}}$ induce the same group isomorphism from $G$ into $\operatorname{PSL}_{2}(\mathbf{C})$.

Since injectivity radii (with respect to the Poincaré metric on $\Delta$ ) of $f_{n}$ are uniformly bounded below (see [18, Lemma 5.1]), for any $z \in \Omega_{0}(G)$, there exist an open neighborhood $U$ of $z$ and suitable branches of the inverse maps $f_{n}{ }^{-1}$ and $f^{-1}$ on $U$ such that $f_{n}^{-1}$ converges to $f^{-1}$ uniformly on $U$. Hence one can see that $\hat{\mu}_{n}$ converges to $\hat{\mu}$ locally uniformly on $\Omega_{0}(G)$. Therefore, $w_{\hat{\mu}_{n}} \circ\left(w_{\hat{\mu}}\right)^{-1}$ converges to a conformal map $w_{\infty} \circ\left(w_{\hat{\mu}}\right)^{-1}$ locally uniformly on $w_{\hat{\mu}}\left(\Omega_{0}(G)\right)$, and hence, the Beltrami coefficient of $w_{\infty}$ is equal to $\hat{\mu}$ almost everywhere on $\Omega_{0}(G)$. Since there is no essential deformation on $\Omega(G)-\Omega_{0}(G)$ by assumption and on $\Lambda(G)$ by Sullivan's rigidity theorem [27], $w_{\infty}$ and $w_{\hat{\mu}}$ induce the same group isomorphism.

Corollary 3.2. Let $[\rho, G]$ be an element of $C_{X}$ such that all components of $\Omega(G) / G$ except for $X=\Omega_{0}(G) / G$ have no moduli of deformation. Then the action of $\operatorname{Mod}(S)$ is continuous at $[\rho]$; that is, if $\left[\rho_{n}\right] \rightarrow[\rho]$ in $C_{X}$ then $\sigma\left(\left[\rho_{n}\right]\right) \rightarrow \sigma([\rho])$ for all $\sigma \in \operatorname{Mod}(S)$.

Proof. By Proposition 3.1, $\mathrm{qc}\left(\left[\rho_{n}\right], \sigma^{-1} X\right) \rightarrow \mathrm{qc}\left([\rho], \sigma^{-1} X\right)$ for all $\sigma \in \operatorname{Mod}(S)$. Therefore, $\sigma\left(\left[\rho_{n}\right]\right)=\mathrm{qc}\left(\left[\rho_{n}\right], \sigma^{-1} X\right) \circ \sigma_{*}^{-1}$ converges algebraically to $\sigma([\rho])=$ $\mathrm{qc}\left([\rho], \sigma^{-1} X\right) \circ \sigma_{*}{ }^{-1}$.

Remark. In [13], Kerckhoff and Thurston showed that there is a Bers slice $B_{X}$ and a point $[\rho] \in \partial B_{X}$ at which the action $\bmod (S)$ is not continuous.

\section{Thurston's compactness theorem}

In this section, we introduce Thurston's compactness theorem [28], which will play an important role in the following sections.

Let $M$ be a compact 3-manifold with boundary $\partial M$. A non-trivial closed curve $\gamma$ on $\partial M$ is said to be compressible if it is null homotopic in $M$; otherwise it is incompressible. A proper map $f:(A, \partial A) \rightarrow(M, \partial M)$ of an annulus $A$ into $M$ is said to 
be essential if $f_{*}: \pi_{1}(A) \rightarrow \pi_{1}(M)$ is an injection and $f$ is not homotopic (as a map of pairs) to a map into $\partial M$.

Definition. Let $M$ be a compact 3-manifold with boundary $\partial M$. Let $\lambda$ be a system of non-trivial, mutually disjoint, homotopically distinct simple closed curves on $\partial M$. Then a pair $(M, \lambda)$ is doubly incompressible if

(1) every compressible simple closed curve on $\partial M$ intersects $\lambda$ at least three times,

(2) there are no essential annuli with boundary in $\partial M-\lambda$, and

(3) every maximal abelian subgroup of $\pi_{1}(\partial M-\lambda)$ is mapped to a maximal abelian subgroup of $\pi_{1}(M)$.

Let $M$ be as above. In addition, we assume that the interior of $M$ admits a hyperbolic structure. We denote by $A H(M)$ the space of conjugacy classes $[\rho, G]$ of discrete faithful representations $\rho: \pi_{1}(M) \rightarrow G \subset \mathrm{PSL}_{2}(\mathbf{C})$. The space $A H(M)$ is equipped with the algebraic topology. Let $\gamma$ be an incompressible closed curve on $\partial M$. For $[\rho, G] \in A H(M)$, length ${ }_{\rho}(\gamma)$ denotes the length of the geodesic representative of $\gamma$ in the hyperbolic manifold $\mathbf{H}^{3} / G$ if $\rho(\gamma)$ is loxodromic and length $\rho(\gamma)=0$ if $\rho(\gamma)$ is parabolic. For a positive constant $K>0$, we denote by $A H(M, \lambda, K)$ the set of elements $[\rho, G] \in A H(M)$ such that length $(\lambda) \leq K$, where length ${ }_{\rho}(\lambda)$ is the total sum of the lengths of all components of $\lambda$.

Now we can state Thurston's compactness theorem:

Theorem 4.1 (Thurston [28]). Let $M$ be a compact 3-manifold with boundary $\partial M$ whose interior $\operatorname{int}(M)$ admits a hyperbolic structure. If $(M, \lambda)$ is doubly incompressible, then $A H(M, \lambda, K)$ is compact for all $K>0$.

Let $S$ be an oriented compact surface possibly with boundary $\partial S$. A curve system $\lambda=\left\{\alpha_{j}\right\}_{j=1}^{N}$ on $S$ is called homotopically independent if it has the following properties: (1) each $\alpha_{j}$ is a simple closed curve on $S$ and $\alpha_{i} \cap \alpha_{j}=\emptyset$ for $i \neq j$, (2) each $\alpha_{j}$ is non-trivial and not freely homotopic to a component of $\partial S$, and (3) $\alpha_{i}$ is not freely homotopic to $\alpha_{j}$ if $i \neq j$. A homotopically independent curve system $\lambda=\left\{\alpha_{j}\right\}_{j=1}^{N}$ on $S$ is maximal if it divides $S$ into pairs of pants. (If $S$ is a closed surface of genus $g$ with $n$ open discs removed, then $N=3 g-3+n$.) A pair $\left(\lambda, \lambda^{\prime}\right)$ of maximal curve systems on $S$ is said to be binding $S$ if they have no curves in common and if each component of $S-\left(\lambda \cup \lambda^{\prime}\right)$ is a simply connected domain or an annulus containing a component of $\partial S$ in its boundary after realizing $\lambda$ and $\lambda^{\prime}$ by geodesics for a hyperbolic structure on $S$.

The following lemma is discussed in a more general setting in Ohshika [25].

Lemma 4.2. Let $S$ be an oriented compact surface possibly with boundary $\partial S$. Let $\left(\lambda^{\prime}, \lambda^{\prime \prime}\right)$ be a pair of maximal curve systems which binds $S$. For this pair, we de- 
fine a maximal curve system $\lambda$ on $\partial(S \times I)$, where $I$ is the closed interval [0,1], as

$$
\lambda=\left(\lambda^{\prime} \times\{0\}\right) \cup\left(\lambda^{\prime \prime} \times\{1\}\right) \cup\left(\partial S \times\left\{\frac{1}{2}\right\}\right) .
$$

Then $(S \times I, \lambda)$ is doubly incompressible.

Proof. We only consider the case $\partial S \neq \emptyset$, since the proof of the case $\partial S=\emptyset$ is easier. If $\partial S \neq \emptyset$, then $S \times I$ is homeomorphic to a handlebody $H_{g}$ of some genus $g$. We identify $S \times I$ with $H_{g}$ via this homeomorphism. We first check the condition (1) in the definition of double incompressibility. Let $\gamma$ be a compressible simple closed curve on $\partial H_{g}$. Since $S \times\{0\}$ and $S \times\{1\}$ contain no compressible curves, $\gamma$ must intersect a component of $\partial S \times\{1 / 2\}$. If $i(\gamma, \lambda) \leq 2$ (here $i(\cdot, \cdot)$ denotes the geometric intersection number), one can easily see that there exists a component $\delta$ of $\partial S \times\{1 / 2\}$ and a component $W$ of $\partial H_{g}-\left(\left(\lambda^{\prime} \times\{0\}\right) \cup\left(\lambda^{\prime \prime} \times\{1\}\right)\right)$ homeomorphic to a four-timepunctured sphere such that $\gamma \cup \delta \subset W$ and $i(\gamma, \delta)=2$. Let $\alpha$ and $\beta$ be components of $\partial W$ such that $\alpha, \beta$ and $\gamma$ bound a pair of pants $T$. Then, after choosing suitable orientations of $\alpha, \beta$ and $\gamma$, we have $[\gamma]=[\alpha]+[\beta]$ in $H_{1}\left(\partial H_{g}, \mathbf{Z}\right)$, where $[\gamma]$ is the homology class of $\gamma$ and so on. Since $\left(\lambda^{\prime}, \lambda^{\prime \prime}\right)$ binds $S,\langle[\alpha],[\beta]\rangle$ are rank 2 free abelian subgroup of $H_{1}\left(\partial H_{g}, \mathbf{Z}\right)$ which is mapped into $H_{1}\left(H_{g}, \mathbf{Z}\right)$ injectively. This contradicts the assumption that $\gamma$ is null homotopic and hence null homologous.

Now we check the condition (2). Suppose that there exists an essential annulus $f:(A, \partial A) \rightarrow\left(H_{g}, \partial H_{g}\right)$ with boundary in $\partial H_{g}-\lambda$. Let $\gamma$ and $\gamma^{\prime}$ be the components of the image of $\partial A$ in $\partial H_{g}$. Since $\left(\gamma \cup \gamma^{\prime}\right) \cap(\partial S \times\{1 / 2\})=\emptyset, \gamma$ and $\gamma^{\prime}$ may be assumed to be contained in $(S \times\{0\}) \cup(S \times\{1\})$. Let $p: S \times I \rightarrow S$ be the canonical retraction. Then $p(\gamma)$ is homotopic to $p\left(\gamma^{\prime}\right)$ in $S$. Since $\left(\lambda^{\prime}, \lambda^{\prime \prime}\right)$ binds $S$, both $\gamma$ and $\gamma^{\prime}$ are contained in $S \times\{0\}$ or $S \times\{1\}$. Now the retraction above gives a homotopy between $f$ and a map into $\partial H_{g}$. This is a contradiction.

Finally, we check the condition (3). Since all non-trivial abelian subgroups of $\pi_{1}\left(\partial H_{g}-\lambda\right)$ or $\pi_{1}\left(H_{g}\right)$ are isomorphic to $\mathbf{Z}$, we have only to show that all primitive elements of $\pi_{1}\left(\partial H_{g}-\lambda\right)$ are also primitive in $\pi_{1}\left(H_{g}\right)$. This follows from the fact that $H_{g}$ is homotopically equivalent to $S$ by the retraction.

\section{Orbit density for maximal cusps}

Let $[\rho] \in \hat{B}_{X}$. The accidental parabolic locus of $[\rho]$ is a homotopically independent curve system $\lambda=\left\{\alpha_{j}\right\}$ on $S$ such that $\rho\left(\alpha_{j}\right)$ is (the conjugacy class of) a parabolic element of $G=\rho\left(\pi_{1}(S)\right)$ for every $j$, and no simple closed curve which is not homotopic to a component of $\lambda$ has this property. For $[\rho] \in \hat{B}_{X}$, its accidental parabolic locus is uniquely determined up to homotopy. An element $[\rho] \in \hat{B}_{X}$ is called a maximal cusp if its accidental parabolic locus is maximal. It is a well known fact that every maximal cusp is contained in $\partial B_{X}$ and that, for any maximal curve system $\lambda$ on $S$, there exists a unique maximal cusp whose accidental parabolic locus 
is $\lambda$ (see Abikoff [1] and Maskit [20]).

For a simple closed curve $\alpha$ on $S$, let $D_{\alpha} \in \operatorname{Mod}(S)$ denote the Dehn twist once around $\alpha$.

Proposition 5.1. Let $\left(\lambda^{\prime}, \lambda^{\prime \prime}\right)$ be a binding pair of maximal curve systems on S. Let $[\rho] \in \partial B_{X}$ be a maximal cusp whose accidental parabolic locus is $\lambda^{\prime \prime}$. Put $\sigma=D_{\alpha_{1}} \circ \cdots \circ D_{\alpha_{N}} \in \operatorname{Mod}(S)$, where $\lambda^{\prime}=\left\{\alpha_{j}\right\}_{j=1}^{N}$. Then the sequence $\left\{\sigma^{n}([\rho])\right\}_{n \in \mathbf{Z}}$ converges to the maximal cusp $\left[\rho_{\infty}\right] \in \partial B_{X}$ whose accidental parabolic locus is $\lambda^{\prime}$ as $|n| \rightarrow \infty$.

In the proof of Proposition 5.1, we will make use of the following two lemmas; the first one is due to Canary [6] and the second one is a well known technical lemma.

Lemma 5.2 (Canary [6]). Given $A>0$, there exists a constant $R>0$ such that if $G$ is a non-elementary, torsion-free Kleinian group such that every incompressible closed geodesic on $\Sigma=\Omega(G) / G$ has hyperbolic length at least $A$, then for any closed curve $\gamma$ on $\Sigma$,

$$
\text { length }_{N}(\gamma) \leq R \cdot \text { length }_{\Sigma}(\gamma)
$$

where length ${ }_{N}(\gamma)$ and length ${ }_{\Sigma}(\gamma)$ are hyperbolic lengths of geodesic representatives of $\gamma$ in $N=\mathbf{H}^{3} / G$ and in $\Sigma$, respectively.

Lemma 5.3. Let $F_{2}$ be a rank 2 free group and let $\left\{\chi_{n}: F_{2} \rightarrow \operatorname{PSL}_{2}(\mathbf{C})\right\}$ be a sequence of discrete faithful representations which converges algebraically to $\chi_{\infty}$. If a sequence $\left\{\chi_{n}^{\prime}=\psi_{n} \cdot \chi_{n} \cdot \psi_{n}^{-1}\right\}$ also converges algebraically to $\chi_{\infty}^{\prime}$ for a sequence $\left\{\psi_{n}\right\}$ in $\mathrm{PSL}_{2}(\mathbf{C})$, then $\psi_{n}$ converges to some element $\psi_{\infty}$ in $\mathrm{PSL}_{2}(\mathbf{C})$.

Proof of Proposition 5.1. Our argument is almost parallel to that of Kerckhoff and Thurston [13] (see also [5]).

Since $C_{X}$ is compact, the sequence $\left\{\left[\rho_{n}\right]=\sigma^{n}([\rho])\right\}_{n \in \mathbf{Z}}$ has a convergent subsequence. We also denote this subsequence by the same symbol. In fact, in the following argument, we can see that any convergent subsequence of $\left\{\left[\rho_{n}\right]\right\}_{n \in \mathbf{Z}}$ converges to a unique maximal cusp $\left[\rho_{\infty}\right]$, and hence $\left\{\left[\rho_{n}\right]\right\}_{n \in \mathbf{Z}}$ converges without passing to a subsequence.

On the other hand, we shall show that the sequence $\left\{\left[\bar{\rho}_{n}\right]=\mathrm{qc}\left([\rho], \sigma^{-n} X\right)\right\}_{n \in \mathbf{Z}}$ also has a convergent subsequence. Recall that $A H(S \times I)$ is the space of conjugacy classes of discrete faithful representations $\chi: \pi_{1}(S \times I) \rightarrow \mathrm{PSL}_{2}(\mathbf{C})$. We denote by $A H_{\partial S}(S \times I)$ the set of representations $[\chi] \in A H(S \times I)$ such that $\chi(g)$ are parabolic for all $g \in \pi_{1}(\partial S \times I)$. Then we can regard $A H_{\partial S}(S \times I)$ as a subset of the representation space $R(S)$. Now we have a sequence $\left\{\left[\bar{\rho}_{n}\right]\right\}_{n \in \mathbf{Z}}$ in $A H_{\partial S}(S \times I) \subset R(S)$. Let $\lambda$ be the 
maximal curve system on $\partial(S \times I)$ defined by

$$
\lambda=\left(\lambda^{\prime} \times\{0\}\right) \cup\left(\lambda^{\prime \prime} \times\{1\}\right) \cup\left(\partial S \times\left\{\frac{1}{2}\right\}\right) .
$$

Then $(S \times I, \lambda)$ is doubly incompressible by Lemma 4.2. We can see that the sequence $\left\{\left[\bar{\rho}_{n}\right]\right\}_{n \in \mathbf{Z}}$ is contained in $A H(S \times I, \lambda, K)$ for some $K>0$ since we have

$$
\text { length }_{\bar{\rho}_{n}}\left(\lambda^{\prime \prime} \times\{1\}\right)=\text { length }_{\bar{\rho}_{n}}\left(\partial S \times\left\{\frac{1}{2}\right\}\right)=0
$$

and

$$
\text { length }_{\bar{\rho}_{n}}\left(\lambda^{\prime} \times\{0\}\right) \leq R \cdot \text { length }{ }_{\sigma^{-n} X}\left(\lambda^{\prime} \times\{0\}\right)=R \cdot \text { length }_{X}\left(\lambda^{\prime} \times\{0\}\right)
$$

from Lemma 5.2. Since $A H(S \times I, \lambda, K)$ is a compact subset in $A H(S \times I)$ by Theorem 4.1, we have a convergent subsequence of $\left\{\left[\bar{\rho}_{n}\right]\right\}_{n \in \mathbf{Z}}$ in $A H(S \times I)$ and hence in $A H_{\partial S}(S \times I) \subset R(S)$. Again, we denote this subsequence by the same symbol.

Take representatives $\rho_{n}$ of $\left[\rho_{n}\right]=\sigma^{n}([\rho])$ so that the sequence $\left\{\rho_{n}\right\}$ converges to a representation $\rho_{\infty}$. Since $\left[\rho_{n}\right]=\sigma^{n}([\rho])=\mathrm{qc}\left([\rho], \sigma^{-n} X\right) \circ \sigma_{*}^{-n}=\left[\bar{\rho}_{n}\right] \circ \sigma_{*}^{-n}$, we may assume that $\bar{\rho}_{n}=\rho_{n} \circ \sigma_{*}^{n}$. In addition, there are elements $\psi_{n} \in \mathrm{PSL}_{2}(\mathbf{C})$ such that the sequence $\left\{\psi_{n} \cdot \bar{\rho}_{n} \cdot \psi_{n}{ }^{-1}\right\}$ converges to a representation $\bar{\rho}_{\infty}$, since $\left\{\left[\bar{\rho}_{n}\right]\right\}_{n \in \mathbf{Z}}$ is a convergent sequence.

Now let $\alpha$ be a component of $\lambda^{\prime}$. We are going to show that $\rho_{\infty}(\alpha)$ is a parabolic element. Let $T$ be a component of $S-\lambda^{\prime}$ containing $\alpha$ in its boundary and $\alpha^{\prime}(\neq \alpha)$ be a component of $\lambda^{\prime}$ or a component of $\partial S$ contained in the boundary of $T$. Choose a base point $x$ in $T$ and regard $\pi_{1}(S)=\pi_{1}(S, x)$. By abuse of notation, $\alpha$ and $\alpha^{\prime}$ also denote the elements of $\pi_{1}(S, x)$ freely homotopic to $\alpha$ and $\alpha^{\prime}$ respectively. Moreover, we assume that (the representatives of) $\alpha, \alpha^{\prime} \in \pi_{1}(S, x)$ contained in $T$. Note that $\left\langle\alpha_{1}, \alpha_{2}\right\rangle$ is a rank 2 free subgroup of $\pi_{1}(S, x)$. Since $\overline{\rho_{n}}(\alpha)=\rho_{n} \circ \sigma_{*}^{n}(\alpha)=\rho_{n}(\alpha)$ and $\overline{\rho_{n}}\left(\alpha^{\prime}\right)=\rho_{n} \circ \sigma_{*}^{n}\left(\alpha^{\prime}\right)=\rho_{n}\left(\alpha^{\prime}\right)$, the elements $\psi_{n} \in \mathrm{PSL}_{2}(\mathbf{C})$ may be taken to be the identity by Lemma 5.3.

One can find non-trivial elements $\gamma_{1}, \gamma_{2} \in \pi_{1}(S, x)$ which satisfy the following conditions (see Fig. 2): (1) $\gamma_{j}$ intersects $\alpha$ twice in the opposite direction for $j=1,2$, (2) $\gamma_{j}$ does not intersect any other components of $\lambda^{\prime}$ for $j=1,2$, and (3) $\left\langle\gamma_{1}, \gamma_{2}\right\rangle$ is a rank 2 free subgroup of $\pi_{1}(S, x)$.

Then we have

$$
\left\{\begin{array}{c}
\bar{\rho}_{n}\left(\gamma_{1}\right)=\rho_{n}\left(\alpha^{n}\right) \cdot \rho_{n}\left(\gamma_{1}\right) \cdot \rho_{n}\left(\alpha^{-n}\right), \\
\bar{\rho}_{n}\left(\gamma_{2}\right)=\rho_{n}\left(\alpha^{n}\right) \cdot \rho_{n}\left(\gamma_{2}\right) \cdot \rho_{n}\left(\alpha^{-n}\right) .
\end{array}\right.
$$

Since both the sequences $\left\{\rho_{n}\right\}$ and $\left\{\bar{\rho}_{n}\right\}$ are convergent sequence, Lemma 5.3 again implies that $\rho_{n}\left(\alpha^{n}\right)$ converges to an element $\hat{\alpha}$ in $\operatorname{PSL}_{2}(\mathbf{C})$. Since $\rho_{n}(\alpha)$ commutes with $\rho_{n}\left(\alpha^{n}\right)$ for all $n, \rho_{\infty}(\alpha)$ commutes with $\hat{\alpha}$. If the abelian subgroup $\left\langle\rho_{\infty}(\alpha), \hat{\alpha}\right\rangle$ were 

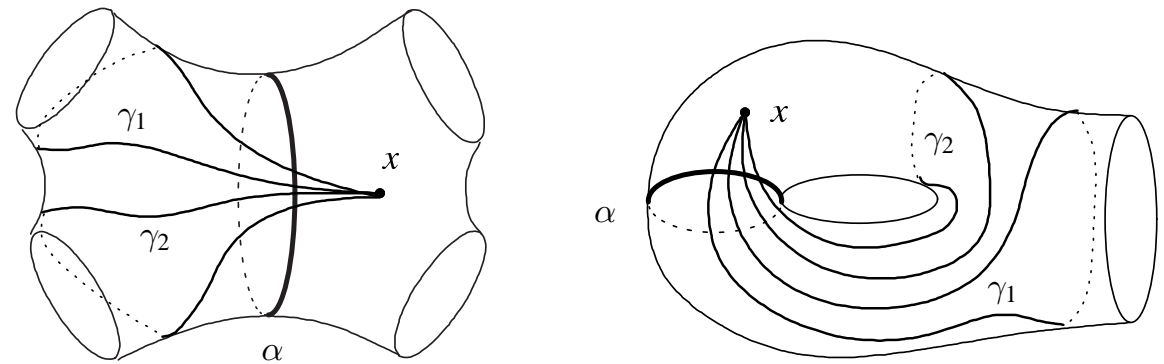

Fig. 2. $\quad \gamma_{1}$ and $\gamma_{2}$

isomorphic to $\mathbf{Z}$, then $\rho_{\infty}\left(\alpha^{k}\right)=\hat{\alpha}^{l}$ for some integers $k$ and $l$, and thus $\rho_{n}\left(\alpha^{n l-k}\right) \rightarrow$ id. This contradicts the fact that $\left[\rho_{n}\right]$ are discrete faithful representations (cf. Jørgensen [12, Lemma 2]). Therefore we conclude that $\left\langle\rho_{\infty}(\alpha), \hat{\alpha}\right\rangle$ is isomorphic to $\mathbf{Z} \oplus \mathbf{Z}$, and hence it is a rank 2 parabolic subgroup in $\operatorname{PSL}_{2}(\mathbf{C})$. In particular, $\rho_{\infty}(\alpha)$ is parabolic. The same argument works well for all components of $\lambda^{\prime}$. Therefore we can conclude that $\left[\rho_{\infty}\right]$ is a maximal cusp whose accidental parabolic locus is $\lambda^{\prime}$.

Lemma 5.4. For any two maximal curve systems $\lambda=\left\{\alpha_{j}\right\}_{j=1}^{N}$ and $\lambda^{\prime}=\left\{\beta_{j}\right\}_{j=1}^{N}$ on $S$, there exists a maximal curve system $\nu=\left\{\gamma_{j}\right\}_{j=1}^{N}$ such that the pairs $(\lambda, \nu)$ and $\left(\nu, \lambda^{\prime}\right)$ are binding $S$.

Proof. There exists a simple closed curve $\delta$ on $S$ such that $i\left(\delta, \alpha_{j}\right)>0$ for all $j$ (see [8]). Put $\sigma=D_{\alpha_{1}} \circ \cdots \circ D_{\alpha_{N}}$. If $i\left(\beta_{j}, \lambda\right)=0$ then $\beta_{j}=\alpha_{i}$ for some $i$ and hence $i\left(\beta_{j}, \sigma^{n}(\delta)\right)>0$ for all $n$. If $i\left(\beta_{j}, \lambda\right)>0$ then $i\left(\beta_{j}, \alpha_{i}\right)>0$ for some $i$. In this case, $i\left(\beta_{j}, \sigma^{n}(\delta)\right)>0$ for all but finitely many $n$. Therefore, for sufficiently large $n$, $i\left(\beta_{j}, \sigma^{n}(\delta)\right)>0$ holds for all $j$. Fix such $n$ and let $\gamma_{1}=\sigma^{n}(\delta)$. Choose simple closed curves $\gamma_{2}, \ldots, \gamma_{N}$ so that $\nu=\left\{\gamma_{j}\right\}_{j=1}^{N}$ is a maximal curve system. This $\nu$ satisfies the desired condition.

It was shown by McMullen [24] that the set of maximal cusps is dense in $\partial B_{X}$. Since the number of ways to decompose $S$ into pairs of pants up to the action of $\operatorname{Mod}(S)$ is finite, the set of maximal cusps in $\partial B_{X}$ decomposes into finitely many orbits under the action of $\operatorname{Mod}(S)$. The next theorem shows that each orbit is dense in $\partial B_{X}$.

Theorem 5.5. For any maximal cusp $[\rho] \in \partial B_{X}$, its orbit $\{\sigma([\rho])\}_{\sigma \in \operatorname{Mod}(S)}$ under the action of $\operatorname{Mod}(S)$ is dense in $\partial B_{X}$.

Proof. Since the set of maximal cusps is dense in $\partial B_{X}$, we have only to show that, for arbitrary fixed two maximal cusps $[\rho]$ and $\left[\rho^{\prime}\right]$ in $\partial B_{X}$, the orbit 
$\{\sigma([\rho])\}_{\sigma \in \operatorname{Mod}(S)}$ of $[\rho]$ contains a sequence converging to $\left[\rho^{\prime}\right]$. Let $\lambda$ and $\lambda^{\prime}$ be accidental parabolic loci for $[\rho]$ and $\left[\rho^{\prime}\right]$, respectively. Then we can find a maximal curve system $\nu=\left\{\gamma_{j}\right\}_{j=1}^{N}$ such that both the pairs $(\lambda, \nu)$ and $\left(\nu, \lambda^{\prime}\right)$ are binding $S$ (Lemma 5.4). Put $\sigma=D_{\gamma_{1}} \circ \cdots \circ D_{\gamma_{N}}$ and $\tau=D_{\beta_{1}} \circ \cdots \circ D_{\beta_{N}}$, where $\lambda^{\prime}=\left\{\beta_{j}\right\}_{j=1}^{N}$. Then $\sigma^{n}([\rho])$ converges to a maximal cusp $\left[\rho^{\prime \prime}\right] \in \partial B_{X}$ whose accidental parabolic locus is $\nu$ by Proposition 5.1. Similarly $\tau^{n}\left(\left[\rho^{\prime \prime}\right]\right)$ converges to $\left[\rho^{\prime}\right]$. Since the action of $\operatorname{Mod}(S)$ is continuous at maximal cusps (Corollary 3.2), we can find a desired sequence by a diagonal argument.

\section{Orbits of Schottky groups and Bers boundary}

In this section, we assume that $S$ is a closed surface of genus $\geq 2$. We denote by $S_{X}$ the set of $[\rho, G] \in C_{X}$ such that $G$ is a Schottky group. The aim of this section is to prove the following theorem.

Theorem 6.1. Let $S$ be a closed surface of genus $\geq 2$. For any $X \in T(S)$, the set of accumulation points of $S_{X}$ contains the boundary $\partial B_{X}$ of the Bers slice $B_{X}$.

REMARK. It is known by Gallo [9] that there is an accumulation point of $S_{X}$ which is not contained in $\partial B_{X}$. This fact can be seen also from a slight modification of the proof of the above theorem.

In the proof of Theorem 6.1, we need the following lemma which give a sufficient condition for an element $[\rho] \in C_{X}$ to be contained in $\partial B_{X}$.

Lemma 6.2. Let $S$ be a compact surface of hyperbolic type possibly with boundary. Let $X \in T(S),[\rho] \in C_{X}$ and $\lambda=\left\{\alpha_{j}\right\}$ be a maximal curve system on $S$. If $\rho\left(\alpha_{j}\right)$ are parabolic for all $j$, then $[\rho]$ is the maximal cusp in $\partial B_{X}$ whose accidental parabolic locus is $\lambda$.

Proof. We have only to show that $[\rho]$ is a faithful representation. Suppose that $\rho: \pi_{1}(S) \rightarrow G$ is not faithful. Then the covering map $p: \Omega_{0}(G) \rightarrow X=\Omega_{0}(G) / G$ is not universal, where $\Omega_{0}(G)$ is the unique invariant component of $G$. Then, by the planarity theorem (see [22], X.A.4), there exist a non-trivial simple closed curve $\delta$ on $X$ and a simple closed curve $\tilde{\delta}$ on $\Omega_{0}(G)$ such that $p \mid \tilde{\delta}: \tilde{\delta} \rightarrow \delta$ is a finite-sheeted covering map; say $k$-sheeted. Let $g \in G$ be a generator for the stabilizer of $\tilde{\delta}$ in $G$. Since $\lambda(\subset X)$ is maximal and $\delta$ is not parallel to a component of $\lambda$, it follows that $\delta$ must intersect some component of $\lambda$, say $\alpha_{1}$. We may assume that the number of intersection points of $\delta$ and $\alpha_{1}$ is equal to $i\left(\delta, \alpha_{1}\right)$. Let $\tilde{\alpha}_{1}$ be a connected component of $p^{-1}\left(\alpha_{1}\right)$ on $\Omega_{0}(G)$ which intersects $\tilde{\delta}$. Let $h$ be a parabolic element which is conjugate to $\rho\left(\alpha_{1}\right)$ in $G$ and stabilizing $\tilde{\alpha}_{1}$. By adjoining the fixed point of $h$ to $\tilde{\alpha}_{1}$, we obtain a simple closed curve, which divides $\hat{\mathbf{C}}$ into two domains. Let $D$ be one of the 
two domains such that $D$ satisfies $D \cap g(D)=\emptyset$ if $k>1$. Let $\eta_{1}$ be a connected component of $D \cap \tilde{\delta}$ and $\beta$ be the arc in $\tilde{\alpha}_{1}$ which connects the end points of $\eta_{1}$. Let $\tilde{\delta}_{1}=\eta_{1} \cup \beta$ and let $\tilde{\delta}_{2}$ be the closed curve $\tilde{\delta}$ with $\eta_{1}, g\left(\eta_{1}\right), \ldots, g^{k-1}\left(\eta_{1}\right)$ replaced by $\beta, g(\beta), \ldots, g^{k-1}(\beta)$. Then, for $j=1,2, \tilde{\delta}_{j}$ projects to a simple closed curve $\delta_{j}$ on $X$ such that $p \mid \tilde{\delta}_{j}: \tilde{\delta}_{j} \rightarrow \delta_{j}$ is a finite-sheeted covering map. Moreover, note that $i\left(\delta_{j}, \lambda\right)$ is strictly less than $i(\delta, \lambda)$ for $j=1,2$. Since $\delta$ is non-trivial and $\delta=\delta_{1} \cdot \delta_{2}$, either $\delta_{1}$ or $\delta_{2}$ are non-trivial. After a finite number of steps as above, we obtain a non-trivial simple closed curve $\delta^{\prime}$ such that $i\left(\delta^{\prime}, \lambda\right)=0$ and that, for a connected component $\tilde{\delta}^{\prime}$ of $p^{-1}\left(\delta^{\prime}\right), p \mid \tilde{\delta}^{\prime}: \tilde{\delta}^{\prime} \rightarrow \delta^{\prime}$ is a finite-sheeted covering map. This is a contradiction.

Proof of Theorem 6.1. Let $S$ be a closed surface of genus $g \geq 2$. Let $[\rho, G]$ be an element of $S_{X}$. We claim that there exists an element $\sigma \in \operatorname{Mod}(S)$ such that the sequence $\left\{\sigma^{n}([\rho])\right\}_{n \in \mathbf{Z}}$ converges to some maximal cusp $\left[\rho_{\infty}\right] \in \partial B_{X}$ as $|n| \rightarrow \infty$. If it has been shown, the similar argument in Theorem 5.5 reveals that the claim of the theorem holds: In fact, for any element $\left[\rho^{\prime}\right] \in \partial B_{X}$, there exists a sequence $\left\{\tau_{n}\right\}$ in $\operatorname{Mod}(S)$ such that $\tau_{n}\left(\left[\rho_{\infty}\right]\right)$ converges to $\left[\rho^{\prime}\right]$ by Theorem 5.5. Since the action of $\operatorname{Mod}(S)$ is continuous at maximal cusps (Corollary 3.2), we can find a sequence in $S_{X}$ which converges to $\left[\rho^{\prime}\right]$ by a diagonal argument.

Now we will show that there exists an element $\sigma \in \operatorname{Mod}(S)$ such that the sequence $\left\{\sigma^{n}([\rho])\right\}_{n \in \mathbf{Z}}$ converges to some maximal cusp $\left[\rho_{\infty}\right] \in \partial B_{X}$ as $|n| \rightarrow \infty$. (Most of the following argument is similar to that of the proof of Proposition 5.1.) Note that the Kleinian manifold $N_{G}=\left(\mathbf{H}^{3} \cup \Omega(G)\right) / G$ is homeomorphic to a handlebody $H_{g}$ of genus $g$ whose boundary $\partial H_{g}$ is homeomorphic to $S$. Under the identification $G=\pi_{1}\left(H_{g}\right)$, we have a proper embedding $\Psi: A H\left(H_{g}\right) \rightarrow R(S)$ where $\Psi$ maps the conjugacy class of $\chi: \pi_{1}\left(H_{g}\right) \rightarrow \mathrm{PSL}_{2}(\mathbf{C})$ to the conjugacy class of $\chi \circ \rho: \pi_{1}(S) \rightarrow G=\pi_{1}\left(H_{g}\right) \rightarrow \mathrm{PSL}_{2}(\mathbf{C})$. By identifying $A H\left(H_{g}\right)$ with its image $\Psi\left(A H\left(H_{g}\right)\right)$, we regard $A H\left(H_{g}\right)$ as a subset of $R(S)$.

Let $\Sigma$ be a compact oriented surface with boundary $\partial \Sigma$ such that $\Sigma \times I$ is homeomorphic to $H_{g}$. (For example, let $\Sigma$ be a closed disk with $g$ open disk removed.) We can find a pair $\left(\lambda^{\prime}, \lambda^{\prime \prime}\right)$ of maximal curve systems on $\Sigma$ which binds $\Sigma$ (cf. Lemma 5.4). Using this pair, we define a maximal curve system $\lambda$ on $S=$ $\partial(\Sigma \times I)=\partial H_{g}$, as

$$
\lambda=\left(\lambda^{\prime} \times\{0\}\right) \cup\left(\lambda^{\prime \prime} \times\{1\}\right) \cup\left(\partial \Sigma \times\left\{\frac{1}{2}\right\}\right) .
$$

Then $\left(H_{g}, \lambda\right)$ is doubly incompressible by Lemma 4.2 and hence $A H\left(H_{g}, \lambda, K\right)$ is compact by Theorem 4.1. Put $\sigma=D_{\alpha_{1}} \circ \cdots \circ D_{\alpha_{N}} \in \operatorname{Mod}(S)$, where $\lambda=\left\{\alpha_{j}\right\}_{j=1}^{N}$. Now we consider the sequence $\left\{\left[\bar{\rho}_{n}\right]=\mathrm{qc}\left([\rho], \sigma^{-n} X\right)\right\}_{n \in \mathbf{Z}}$ in $A H\left(H_{g}\right) \subset R(S)$. Then we can see that the sequence $\left\{\left[\bar{\rho}_{n}\right]\right\}_{n \in \mathbf{Z}}$ is contained in a compact set $A H\left(H_{g}, \lambda, K\right) \subset R(S)$ 
for some $K>0$ since we have

$$
\text { length }_{\bar{\rho}_{n}}(\lambda) \leq R \cdot \text { length }_{\sigma^{-n} X}(\lambda)=R \cdot \text { length }_{X}(\lambda) \text {. }
$$

from Lemma 5.2 for some $R>0$. Therefore the sequence $\left\{\left[\bar{\rho}_{n}\right]\right\}_{n \in \mathbf{Z}}$ has a convergent subsequence.

On the other hand, since $C_{X}$ is compact, $\left\{\left[\rho_{n}\right]=\sigma^{n}([\rho])\right\}_{n \in \mathbf{Z}}$ also has a convergent subsequence. Take representatives $\rho_{n}$ of $\left[\rho_{n}\right]=\sigma^{n}([\rho])$ so that the sequence $\left\{\rho_{n}\right\}$ converges to a representation $\rho_{\infty}$. Since $\left[\rho_{n}\right]=\left[\bar{\rho}_{n}\right] \circ \sigma_{*}^{-n}$, we may assume that $\bar{\rho}_{n}=\rho_{n} \circ \sigma_{*}^{n}$. In addition, there are elements $\psi_{n} \in \mathrm{PSL}_{2}(\mathbf{C})$ such that the sequence $\left\{\psi_{n} \cdot \bar{\rho}_{n} \cdot \psi_{n}{ }^{-1}\right\}$ converges to a representation $\bar{\rho}_{\infty}$.

For any component $\alpha$ of $\lambda$, we claim that $\rho_{\infty}(\alpha)$ is a parabolic element. But this can be seen from the same argument in the proof of Proposition 5.1. Therefore, we leave the proof to the reader.

Since $\rho_{\infty}(\alpha)$ are parabolic for any component $\alpha$ of $\lambda$, we can conclude that $\left[\rho_{\infty}\right]$ is a maximal cusp in $\partial B_{X}$ whose accidental parabolic locus is $\lambda$ by Lemma 6.2.

\section{Some property of the set of Schottky groups}

Let $S$ be a closed surface of genus $\geq 2$. In this section, we collect some property of $S_{X}$ which can be easily seen. For a representation $\rho$ of $\pi_{1}(S)$ onto a Kleinian group $G$, we denote by $N_{G}$ its Kleinian manifold $\left(\mathbf{H}^{3} \cup \Omega(G)\right) / G$.

Lemma 7.1. The mapping class group $\operatorname{Mod}(S)$ acts on $S_{X}$ transitively; that is, $S_{X}=\{\sigma([\rho])\}_{\sigma \in \operatorname{Mod}(S)}$ for any $[\rho] \in S_{X}$.

Proof. Let $\left[\rho_{1}, G_{1}\right]$ and $\left[\rho_{2}, G_{2}\right]$ be arbitrary two elements of $S_{X}$. Then there exists a homeomorphism $N_{G_{1}} \rightarrow N_{G_{2}}$ such that the restriction of this map to the boundaries is a quasiconformal map $\Omega_{0}\left(G_{1}\right) / G_{1} \rightarrow \Omega_{0}\left(G_{2}\right) / G_{2}$. Now one can see that $\left[\rho_{2}\right]=\sigma\left(\left[\rho_{1}\right]\right)$, where $\sigma \in \operatorname{Mod}(S)$ is the isotopy class of a homeomorphism of $S$ induced by the quasiconformal map.

A Kleinian group is called geometrically finite if it has a finite sided convex fundamental polyhedron in $\mathbf{H}^{3}$.

Lemma 7.2 (Hejhal [11], Matsuzaki [23]). Each element $[\rho] \in S_{X}$ is an isolated point in $C_{X}$. On the other hand, if a torsion-free, geometrically finite element $[\rho] \in C_{X}$ is isolated in $C_{X}$, then $[\rho] \in S_{X}$.

Proof. The first statement is due to Hejhal [11], who showed that any element $[\rho, G] \in \hat{C}_{X}$ such that $G$ is a Schottky group is isolated in $\hat{C}_{X}$. Conversely, let take 
an element $[\rho] \in C_{X}$ which is isolated in $C_{X}$. Since the same argument of Lemma 2.1 reveals that $\hat{C}_{X}-C_{X}$ is closed, $[\rho]$ is also isolated in $\hat{C}_{X}$. It was shown by Matsuzaki ([23, Theorem 3]) that, if a torsion-free, geometrically finite element $[\rho] \in \hat{C}_{X}$ is isolated in $\hat{C}_{X}$, then $[\rho]$ is a Schottky group. Thus, the second statement is proved.

Remark. In Matsuzaki [23], obtained is a necessary and sufficient condition for a (not necessarily torsion-free) geometrically finite element of $\hat{C}_{X}$ to be isolated in $\hat{C}_{X}$.

For $[\rho] \in S_{X}$, the following lemma gives a characterization of the elements of $\operatorname{Mod}(S)$ which stabilize $[\rho]$.

Lemma 7.3. Let $[\rho, G] \in S_{X}$ and $\sigma \in \operatorname{Mod}(S)$. Then the following are equivalent:

(1) $\sigma([\rho])=[\rho]$,

(2) $\sigma_{*}(\operatorname{ker} \rho)=\operatorname{ker} \rho$, and

(3) $\sigma$ can be extended to a homeomorphism of the Kleinian manifold $N_{G}$, where $\sigma$ is regarded as a homeomorphism of $X=\partial N_{G}$.

Proof. (1) $\Rightarrow$ (2) and (3) $\Rightarrow$ (2) are trivial. (2) $\Rightarrow$ (1) can be seen from Matsuzaki [23, Theorem 2] and Lemma 7.2. We will show that $(2) \Rightarrow(3)$. Let $(f, \rho)$ be the projective structure corresponding to $[\rho]$. We may assume that $\sigma: X \rightarrow X$ is a quasiconformal map. Let $\tilde{\sigma}: \Delta \rightarrow \Delta$ be a lift of $\sigma: X \rightarrow X$. If $\sigma_{*}(\operatorname{ker} \rho)=\operatorname{ker} \rho$, then $\tilde{\sigma}$ descends to a quasiconformal map $\hat{\sigma}: f(\Delta) \rightarrow f(\Delta)$, because the covering group $f: \Delta \rightarrow f(\Delta)$ is $\operatorname{ker} \rho$. Since $G=\rho\left(\pi_{1}(S)\right)$ is geometrically finite and $\Omega(G)=f(\Delta)$, Marden's isomorphism theorem [19] implies that $\hat{\sigma}$ can be extended to a $G$-compatible quasiconformal automorphism of $\hat{\mathbf{C}}$. This quasiconformal map can be extended to a $G$-compatible homeomorphism of $\mathbf{H}^{3} \cup \hat{\mathbf{C}}$, which descends to a homeomorphism of $N_{G}$ (cf. [7]).

\section{References}

[1] W. Abikoff: On boundaries of Teichmüller spaces and on Kleinian groups:III, Acta Math. 134 (1975), 211-234.

[2] L. Bers: Simultaneous uniformization, Bull. Amer. Math. Soc. 66 (1960), 94-97.

[3] L. Bers: On boundaries of Teichmüller spaces and on Kleinian groups: I, Ann. of Math. 91 (1970), 570-600.

[4] L. Bers: The action of the modular group on the complex boundary, Riemann surfaces and related topics: Proceedings of the 1978 Stony Brook Conference, Ann. of Math. Stud. 97, Princeton Univ. Press, Princeton, 1981, 33-52.

[5] J.F. Brock: Iteration of mapping classes on a Bers slice: examples of algebraic and geometric 
limits of hyperbolic 3-manifolds, Lipa's legacy (New York, 1995), Contemporary Math. 211, Amer. Math. Soc., Providence,1997, 81-106.

[6] R.D. Canary: The Poincaré metric and a conformal version of a theorem of Thurston, Duke Math. J. 64 (1991), 349-359.

[7] A. Douady and C.J. Earle: Conformally natural extension of homeomorphisms of the circle, Acta Math. 157 (1986), 23-48.

[8] A. Fathi, F. Raudenbach and V. Poenaru: Travaux de Thurston sur le surfaces, Astérisque 66-67, Société Mathématique de France, Paris, 1979.

[9] D.M. Gallo: Some special limits of Schottky groups, Proc. Amer. Math. Soc. 118 (1993), 877-883.

[10] D.M. Gallo: Schottky groups and the boundary of Teichmüller space: genus 2, The mathematical legacy of Wilhelm Magnus: groups, geometry and special functions (Brooklyn, NY, 1992), Contemporary Math. 169, Amer. Math. Soc., Providence,1994, 283-305.

[11] D.A. Hejhal: On Schottky and Koebe-like uniformizations, Duke Math. J. 55 (1987), 267-286.

[12] T. Jørgensen: On discrete groups of Möbius transformations, Amer. J. Math. 98 (1976), 739-749.

[13] S.P. Kerckhoff and W.P. Thurston: Non-continuity of the action of the modular group at Bers' boundary of Teichmuller space, Invent. Math. 100 (1990), 25-47.

[14] I. Kra: Deformations of Fuchsian groups, Duke Math. J. 36 (1969), 537-546.

[15] I. Kra: A generalization of a theorem of Poincaré, Proc. Amer. Math. Soc. 27 (1971), 299-302.

[16] I. Kra: Deformations of Fuchsian groups, II, Duke Math. J. 38 (1971), 499-508.

[17] I. Kra: On spaces of Kleinian groups, Comm. Math. Helv. 47 (1972), 53-69.

[18] I. Kra and B. Maskit: Remarks on projective structures, Riemann surfaces and related topics: Proceedings of the 1978 Stony Brook Conference, Ann. of Math. Stud. 97, Princeton Univ. Press, Princeton, 1981, 343-359.

[19] A. Marden: The geometry of finitely generated Kleinian groups, Ann. of Math. 99 (1974), 384-462.

[20] B. Maskit: On boundaries of Teichmüller spaces and on kleinian groups: II, Ann. of Math. 91 (1970), 607-639.

[21] B. Maskit: Self-maps of Kleinian groups, Amer. J. Math. 93 (1971), 840-856.

[22] B. Maskit: Kleinian groups, Springer-Verlag, Berlin, 1988.

[23] K. Matsuzaki: Projective structures inducing covering maps, Duke Math. J. 78 (1995), 413-425.

[24] C.T. McMullen: Cusps are dense, Ann. of Math. 133 (1991), 217-247.

[25] K. Ohshika: Geometrically finite Kleinian groups and parabolic elements, Proc. Edinburgh Math. Soc. 41 (1998), 141-159.

[26] J.P. Otal: Sur le bord du prologement de Bers de l'espace de Teichmüller., C.R. Acad. Sci. Paris, 316 (1993), 157-160.

[27] D.P. Sullivan: On the ergodic theory at infinity of an arbitrary discrete group of hyperbolic motions, Riemann surfaces and related topics: Proceedings of the 1978 Stony Brook Conference, Ann. of Math. Stud. 97, Princeton Univ. Press, Princeton, 1981, 465-496.

[28] W.P. Thurston: Hyperbolic structures on 3-manifolds, III: Deformations of 3-manifolds with incompressible boundary, preprint.

Graduate School of Mathematics

Nagoya University

Nagoya 464-8602, Japan

e-mail: itoken@math.nagoya-u.ac.jp 